\title{
Gravity and the Structure of Noncommutative Algebras
}

\author{
M. Burić ${ }^{1}$, T. Grammatikopoulos ${ }^{2}$, \\ J. Madore $^{3}$, G. Zoupanos ${ }^{2}$ \\ ${ }^{1}$ Faculty of Physics, P.O. Box 368, 11001 Belgrade \\ ${ }^{2}$ Physics Department, National Technical University \\ Zografou Campus, GR-157 80 Zografou, Athens \\ ${ }^{3}$ Laboratoire de Physique Théorique \\ Université de Paris-Sud, Bâtiment 211, F-91405 Orsay
}

\begin{abstract}
A gravitational field can be defined in terms of a moving frame, which when made noncommutative yields a preferred basis for a differential calculus. It is conjectured that to a linear perturbation of the commutation relations which define the algebra there corresponds a linear perturbation of the gravitational field. This is shown to be true in the case of a perturbation of Minkowski space-time.
\end{abstract}




\section{Introduction}

It is known that to a noncommutative geometry one can associate in various ways a gravitational field. This can be elegantly done [1, 2] in the imaginary-time formalism and perhaps less so [3] in the real-time formalism. We examine here the inverse problem, that of associating a noncommutative geometry to a given classical field. As concrete examples, one would like to know to what extent it is possible to give a noncommutative extension of the Schwarzschild metric or of a cosmological metric. We would also like to know how many extensions there are and what their properties. There have been examples constructed [4, 5, 6] more-or-less ad hoc; we give here a more systematic analysis by restricting our considerations to the 'semi-classical' theory, retaining only contributions of first-order in the noncommutativity parameter. As a working hypothesis we shall suppose that there is one physical property, which at large scales manifests itself as gravity and at small scales as noncommutativity.

When a noncommutative geometry is considered to lowest order the commutativity relations define a symplectic form; the metric defines also a curvature. We examine the relation between these two structures which are imposed by the requirements of noncommutative geometry. We show that in certain simple situations the field 'almost' determines the structure of the algebra as well as the differential calculus. Over a given algebra there can be many differential calculi but all must satisfy certain consistency conditions before they can be considered as associated to the algebra. We are therefore interested in the cases where these relations determine the field. We shall consider almost exclusively the almost-commutative limit. We shall also briefly consider a sort of modified form of the background-field approximation in which we suppose that to the structure of a noncommutative algebra and associated differential calculus there has been associated a geometric structure and we proceed so to speak by induction to extend the correspondence to a first-order perturbation.

As a measure of noncommmutativity, and to recall the many parallelisms with quantum mechanics, we use the symbol $k$, which will designate the square of a real number whose value could lie somewhere between the Planck length and the proton radius $m_{P}^{-1}$. Although this is never explicitly used we shall think rather of the former and identify $\hbar$ with Newton's constant $G_{N}$ (in units with $\hbar=1$ ). This becomes important when we consider perturbations. We introduce a set $J^{\mu \nu}$ of elements of an associative algebra $\mathcal{A}$ and use them to define commutation relations

$$
\left[x^{\mu}, x^{\nu}\right]=i \hbar J^{\mu \nu}\left(x^{\sigma}\right) .
$$

The $J^{\mu \nu}$ are of course restricted by Jacobi identities; we see below that there are two other natural requirements which also restrict them.

Let $\mu$ be a typical 'large' source mass with 'Schwarzschild radius' $G_{N} \mu$. If noncommutativity is not directly related to gravity then it makes sense to speak of ordinary gravity as the limit $k \rightarrow 0$ with $G_{N} \mu$ non vanishing. On the other hand if noncommutativity and gravity are directly related then both should vanish with $k$. The two 
points of view are not at odds provided one considers (classical) gravity as a purely macroscopic phenomenon, valid only for 'large' masses. We shall use the dimensionless parameter

$$
\epsilon=\hbar \mu^{2}
$$

as a measure of the relative importance of noncommutative effects. We shall also use the WKB formalism to illustrate the close relation which exists between $J^{\mu \nu}$ and the geometry of the wave. The WKB approximation is a classical description of quantum mechanics in the sense that derivations can be identified with the momenta. We shall show that in the presence of the wave Jacobi identities require a modification of the structure of the algebra. Let $\omega$ be the characteristic mass scale associated with the wave. We shall require the inequalities

$$
\sqrt{\hbar} \ll \omega^{-1} \ll \mu^{-1} .
$$

If we consider $k$ to be of the order of the Planck mass then the first inequality states that the Planck mass is 'large'; the second is the definition of what is meant by a 'high-frequency' wave.

The extra momenta $p_{\alpha}$ which must be added to the algebra in order that the derivations be inner stand in duality to the position operators $x^{\mu}$ by the relation

$$
\left[p_{\alpha}, x^{\mu}\right]=e_{\alpha}^{\mu}
$$

The right-hand side of this identity defines the gravitational field. The left-hand side must obey Jacobi identities. These identities yield relations between quantum mechanics in the given curved space-time and the noncommutative structure of the algebra. The three aspects of reality then, the curvature of space-time, quantum mechanics and the noncommutative structure of space-time are intimately connected. We shall consider here the even more exotic possibility that the field equations of general relativity are encoded also in the structure of the algebra so that the relation between general relativity and quantum mechanics can be understood by the relation which each of these theories has with noncommutative geometry.

In spite of the rather lengthy formalism the basic idea is simple. We start with a classical geometry described by a moving frame $\theta^{\alpha}$ and we associate

$$
\theta^{\alpha} \stackrel{\rho}{\longrightarrow} J^{\mu \nu}
$$

to it a noncommutative algebra with generators $x^{\mu}$ and commutation relations (1.1) which we identify with position space. To this algebra we add the extra elements which are necessary in order that the derivations become inner; this is ordinary quantum mechanics. The new element is the fact that if the original algebra describes a curved space-time then Jacobi identities force the extended algebra to be noncommutative. More details of the map (1.5) will be given in Section 4.

Typically one would proceed in three steps. First choose a moving frame to describe a metric. Quantize it by replacing the moving frame by a frame, as described below. The 
important special cases referred to above would include those frames which could be quantized without ordering problems. Finally one looks for a noncommutative algebra consistent with the resulting differential calculus; this is the image of the map (1.5). Let $e_{\alpha}$ be dual to the left-hand side of (1.5). If we quantize as in (1.4) by imposing the rule

$$
e_{\alpha} \mapsto p_{\alpha}
$$

then from the Jacobi identities we find

$$
\left[p_{\alpha}, J^{\mu \nu}\right]=\left[x^{[\mu},\left[p_{\alpha}, x^{\nu]}\right]\right]
$$

If the space is flat and the frame is the canonical flat frame then the right-hand side vanishes and it is possible to consistently choose the expression $J^{\mu \nu}$ to be equal to a constant or even to vanish. But on the other hand, if the space is curved the righthand side cannot vanish identically; we must conclude then that $J^{\mu \nu}$ is non-trivial. This means that the kernel of the map (1.5) must vanish. On the other hand it cannot be single valued for any constant $J$ has flat space as inverse image.

The physical idea we have in mind has been given elsewhere [7, 8, 9]. One can use a solid-state analogy and think of the ordinary Minkowski coordinates as macroscopic order parameters obtained by 'coarse-graining' over regions whose size is determined by a fundamental area scale $k$, which is presumably, but not necessarily, of the order of the Planck area $G \hbar$. They break down and must be replaced by elements of a noncommutative algebra when one considers phenomena on smaller scales. A simple visualization is afforded by the orientation order parameter of nematic liquid crystals. The commutative free energy is singular in the core region of a disclination. There is of course no physical singularity; the core region can simply not be studied using the commutative order parameter.

There is also a certain similarity with the effect of screening in quantum field theory and in plasma physics. One can consider a 'point' as surrounded by a 'cloud of void' which 'screens' it from neighbouring 'points'. Because the commutator defines in the commutative limit an antisymmetric tensor field there are obvious analogies with spin and with the electromagnetic field; we have not however found any particularly fruitful insights using these. In the limit when the gravitational field vanishes there still remains a definite frame at each point defined, for example, by the Petrov vectors. So even in flat space, if considered as the result of such a limiting process, local Lorentz invariance is broken. This residual memory could be considered to be similar to that invoked in Mach's principle. Other reasons have been proposed [10] for this breaking.

A detailed description of the method we shall use has been given in a previous article [11] and it suffices therefore here to outline the prescription. We suppose that a complete consistent noncommutative geometry has been given. By this we mean that the frame and the commutation relations are explicitly known. We shall perturb both the geometry and the algebra and show that the perturbation of the one can be intimately related with that of the other such that the resulting geometry is consistent. 
The fact that the geometry depends only on the formal algebraic structure of the algebra, seemingly independent of the representation is perhaps due to the fact that only first-order perturbations are explicitly calculated. Although one cannot claim to have defined completely an algebra without a choice of state, we have not found it necessary to use a concrete representation in the calculations we have presented here. This is certainly related to the fact that most concrete calculations are presented only in the quasi-classical approximation. Although noncommutative 'gravity' in the Kaluza-Klein sense had been investigated earlier [7, 12] it would seem that the first concrete example of noncommutative 'gravity' was [13] an extension of the 2-sphere. Although not very interesting as a realistic example of gravity it clearly illustrates the relation between the commutation relations and the effective classical gravitational field. There have been several recent investigations of the same subject, at least two of which [14, 15] are not far in spirit from the present calculations.

\section{General considerations}

Let then $\mathcal{A}$ be a noncommutative $*$-algebra generated by four hermitian elements $x^{\mu}$ which satisfy the commutation relations (1.1). Assume that over $\mathcal{A}$ is a differential calculus which is such [9] that the module of 1-forms is free and possesses a preferred frame $\theta^{\alpha}$ which commutes,

$$
\left[x^{\mu}, \theta^{\alpha}\right]=0,
$$

with the algebra. The space one obtains in the commutative limit is therefore parallelizable with a global moving frame $\tilde{\theta}^{\alpha}$ defined to be the commutative limit of $\theta^{\alpha}$. We can write the differential

$$
d x^{\mu}=e_{\alpha}^{\mu} \theta^{\alpha}, \quad e_{\alpha}^{\mu}=e_{\alpha} x^{\mu}
$$

The algebra is defined by a product which is restricted by the matrix of elements $J^{\mu \nu}$; the metric is defined, we shall see below, by the matrix of elements $e_{\alpha}^{\mu}$. Consistency requirements, essentially determined by Leibniz rules, impose relations between these two matrices which in simple situations allow us to find a one-to-one correspondence between the structure of the algebra and the metric. The input of which we shall make the most use is the Leibniz rule

$$
i \hbar e_{\alpha} J^{\mu \nu}=\left[e_{\alpha}^{\mu}, x^{\nu}\right]-\left[e_{\alpha}^{\nu}, x^{\mu}\right]
$$

One can see here a differential equation for $J^{\mu \nu}$ in terms of $e_{\alpha}^{\mu}$. In important special cases the equation reduces to a simple differential equation of one variable.

The relation (2.3) can be written also as Jacobi identities

$$
\left[p_{\alpha},\left[x^{\mu}, x^{\nu}\right]\right]+\left[x^{\nu},\left[p_{\alpha}, x^{\mu}\right]\right]+\left[x^{\mu},\left[x^{\nu}, p_{\alpha}\right]\right]=0
$$

if one introduce the momenta $p_{\alpha}$ associated to the derivation by the relation (1.4). 
Finally, we must insure that the differential is well defined. A necessary condition is that $d\left[x^{\mu}, \theta^{\alpha}\right]=0$. It follows that

$$
d\left[x^{\mu}, \theta^{\alpha}\right]=\left[d x^{\mu}, \theta^{\alpha}\right]+\left[x^{\mu}, d \theta^{\alpha}\right]=e_{\beta}^{\mu}\left[\theta^{\beta}, \theta^{\alpha}\right]-\frac{1}{2}\left[x^{\mu}, C^{\alpha}{ }_{\beta \gamma}\right] \theta^{\beta} \theta^{\gamma} .
$$

We have here introduced the Ricci rotation coefficients $C^{\alpha}{ }_{\beta \gamma}$. We find then that multiplication of 1 -forms must satisfy

$$
\left[\theta^{\alpha}, \theta^{\beta}\right]=\frac{1}{2} \theta_{\mu}^{\beta}\left[x^{\mu}, C^{\alpha}{ }_{\gamma \delta}\right] \theta^{\gamma} \theta^{\delta} .
$$

Consistency requires then that

$$
\theta_{\mu}^{[\beta}\left[x^{\mu}, C_{\gamma \delta}^{\alpha]}\right]=0 .
$$

We have in general three consistency equations which must be satisfied in order to obtain a noncommutative extension. They are the Leibniz rule (2.3), the Jacobi identity and the condition (2.7) on the differential. The first two constraints follow from Leibniz rules but they are not completely independent of the differential calculus since one involves the momentum operators.

To illustrate the importance of the Jacobi identities we mention that they force a modification of the canonical commutation relations and introduce a dependence

$$
\hbar \delta_{\alpha}^{\mu} \mapsto \hbar e_{\alpha}^{\mu}
$$

of Planck's 'constant' on the gravitational field. We mentioned already that if one place the canonical commutator (1.4) in the Jacobi identity with two coordinate and one momentum entry that for this to be consistent the coordinates in general cannot commute.

\section{Linear perturbations of flat space}

If we consider the $J^{\mu \nu}$ of the previous sections as the components of a classical field on a curved manifold then in the limit when the manifold becomes flat the 'equations of motion' are Lorentz invariant. We notice however that in this limit they are also degenerate. In particular solutions of the form (5.7) are unacceptable. To remedy this we suppose that as $e_{\alpha}^{\lambda} \rightarrow e_{0 \alpha}^{\lambda}$ we obtain

$$
J^{\mu \nu} \rightarrow J_{0}^{\mu \nu}, \quad \operatorname{det} J_{0} \neq 0
$$

Were we to choose $e_{0 \alpha}^{\lambda}$ to be a flat frame then the assumption would mean that $J_{0}^{\mu \nu}$ 'spontaneously' breaks Lorentz invariance. Since Lorentz invariance is broken for every non-flat frame by definition, it would be a stronger assumption to suppose that $J_{0}^{\mu \nu}=0$.

We shall now consider fluctuations around a particular given solution to the problem we have set. We suppose that is we have a reference solution comprising a frame $e_{0 \alpha}^{\lambda}=\delta_{\alpha}^{\lambda}$ and a commutation relation $J_{0}^{\mu \nu}$ which we perturb to

$$
J^{\alpha \beta}=J_{0}^{\alpha \beta}+\epsilon I^{\alpha \beta}, \quad e_{\alpha}^{\mu}=\delta_{\beta}^{\mu}\left(\delta_{\alpha}^{\beta}+\epsilon \Lambda_{\alpha}^{\beta}\right) .
$$


In terms of the unknowns $I$ and $\Lambda$ the Jacobi and Leibniz constraints become respectively

$$
\begin{aligned}
& \epsilon_{\lambda \mu \nu \sigma}\left[x^{\lambda}, I^{\mu \nu}\right]=0, \\
& e_{\alpha} I^{\mu \nu}=\left[\Lambda_{\alpha}^{\mu}, x^{\nu}\right]-\left[\Lambda_{\alpha}^{\nu}, x^{\mu}\right] .
\end{aligned}
$$

We now use the fact, well known from quantum mechanics, that when the value of the commutator is a constant then the commutator is a derivative. That is, for any $f$

$$
\left[x^{\lambda}, f\right]=i \hbar J_{0}^{\lambda \sigma} \partial_{\sigma} f+o\left(\epsilon^{2}\right), \quad\left[p_{\alpha}, f\right]=\partial_{\alpha} f+o\left(\epsilon^{2}\right)
$$

The two constraint equations become

$$
\begin{aligned}
& \epsilon_{\lambda \mu \nu \sigma} J^{\lambda \sigma} \partial_{\sigma} I^{\mu \nu}=0, \\
& e_{\alpha} I^{\mu \nu}=\partial_{\sigma} \Lambda_{\alpha}^{[\mu} J_{0}^{\sigma \nu]} .
\end{aligned}
$$

These two equations are the origin of the particularities of our construction, they and the fact that the 'ground-state' value of $J^{\mu \nu}$ is an invertible matrix.

The constraint equations become particularly transparent if one introduce the new unknowns

$$
\hat{I}_{\alpha \beta}=J_{0 \alpha \gamma}^{-1} J_{0 \beta \delta}^{-1} I^{\gamma \delta}, \quad \hat{\Lambda}_{\alpha \beta}=J_{0 \beta \gamma}^{-1} \Lambda_{\alpha}^{\gamma} .
$$

We decompose also $\hat{\Lambda}$ as the sum

$$
\hat{\Lambda}_{\alpha \beta}=\hat{\Lambda}_{\alpha \beta}^{+}+\hat{\Lambda}_{\alpha \beta}^{-}
$$

of a symmetric and antisymmetric term. The constraints become

$$
\begin{aligned}
& e_{\alpha}\left(\hat{I}+\hat{\Lambda}^{-}\right)_{\beta \gamma}+\left(e_{\alpha} \hat{\Lambda}_{\beta \gamma}^{-}+e_{\beta} \hat{\Lambda}_{\gamma \alpha}^{-}+e_{\gamma} \hat{\Lambda}_{\alpha \beta}^{-}\right)=e_{[\beta} \hat{\Lambda}_{\gamma] \alpha}^{+}, \\
& \epsilon^{\alpha \beta \gamma \delta} e_{\alpha}(\hat{I}+2 \hat{\Lambda})_{\beta \gamma}=0 .
\end{aligned}
$$

We introduce

$$
\hat{I}=\frac{1}{2} \hat{I}_{\alpha \beta} \theta^{\alpha} \theta^{\beta}, \quad \hat{\Lambda}^{-}=\frac{1}{2} \hat{\Lambda}_{\alpha \beta}^{-} \theta^{\alpha} \theta^{\beta} .
$$

The constraints simplify to 'cocycle' conditions. If we multiply (3.10) by $\epsilon^{\alpha \beta \gamma \delta}$ we obtain

$$
\epsilon^{\alpha \beta \gamma \delta} e_{\alpha}\left(\hat{I}+4 \hat{\Lambda}^{-}\right){ }_{\beta \gamma}=0
$$

It follows then that

$$
d \hat{\Lambda}^{-}=0, \quad d \hat{I}=0 .
$$

We can rewrite (3.10) as

$$
e_{\alpha}\left(\hat{I}+\hat{\Lambda}^{-}\right)_{\beta \gamma}=e_{[\beta} \hat{\Lambda}_{\gamma] \alpha}^{+} .
$$

This equation has the integrability conditions

$$
e_{\alpha} e_{[\beta} \hat{\Lambda}_{\gamma] \delta}^{+}-e_{\delta} e_{[\beta} \hat{\Lambda}_{\gamma] \alpha}^{+}=0
$$


But the left-hand side is the linearized approximation to the curvature of a metric with components $g_{\mu \nu}+\epsilon \hat{\Lambda}_{\mu \nu}^{+}$. If it vanishes then the perturbation is a derivative; for some 1-form $A$

$$
\hat{\Lambda}_{\beta \gamma}^{+}=\frac{1}{2} e_{(\beta} A_{\gamma)} .
$$

Equation (3.15) becomes therefore

$$
e_{\alpha}\left(\hat{I}+\hat{\Lambda}^{-}-d A\right)_{\beta \gamma}=0
$$

It follows then that for some 2-form $c$ with constant components $c_{\beta \gamma}$

$$
\hat{\Lambda}^{-}=-\hat{I}+d A+c
$$

The remaining constraints are satisfied identically. The most important relation is Equation (3.19) which, in terms of the original 'unhatted' quantities, becomes

$$
\Lambda_{\beta}^{\alpha}=J_{0 \beta \gamma}^{-1} I^{\alpha \gamma}+J_{0}^{\alpha \gamma}\left(c_{\beta \gamma}+e_{\beta} A_{\gamma}\right) .
$$

This condition is much weaker than, but similar to Equation (5.6).

\section{The algebra to geometry map}

We can now be more precise about the map (1.5). Let $\theta^{\alpha}$ be a frame which is a small perturbation of a flat frame and let $J^{\alpha \beta}$ be the frame components of a small perturbation of a constant 'background' $J_{0}$. Us interests the map

$$
I^{\alpha \beta} \stackrel{\sigma}{\longrightarrow} \Lambda_{\beta}^{\alpha}=J_{0 \beta \gamma}^{-1} I^{\alpha \gamma}+J_{0}^{\alpha \gamma}\left(c_{\beta \gamma}+e_{\beta} A_{\gamma}\right) .
$$

We recall that we are considering only first-order fluctuations around a given frame and that these fluctuations are redundently parameterized by the array $\Lambda_{\beta}^{\alpha}$. We can rewrite the map $\rho$ as a map

$$
\Lambda_{\beta}^{\alpha} \stackrel{\rho}{\longrightarrow} I^{\alpha \beta}
$$

It can be defined as an inverse of the map $\sigma$ defined in Equation (4.1).

If we neglect all terms which are gradients then we see that the extension of $\sigma$ to the metric is given by

$$
\left.g^{\alpha \beta}=g_{0}^{\alpha \beta}-\epsilon h^{\alpha \beta}, \quad h^{\alpha \beta}=\Lambda^{(\alpha \beta)}=J_{0}^{(\alpha \gamma} \hat{I}_{\gamma}{ }^{\beta}\right) .
$$

We recall that a perturbation of a frame

$$
e_{\alpha}^{\mu}=e_{0 \beta}^{\mu}\left(\delta_{\alpha}^{\beta}+\epsilon \Lambda_{\alpha}^{\beta}\right)
$$

engenders a perturbation

$$
g^{\mu \nu}=g_{0}^{\mu \nu}-\epsilon h^{\mu \nu}, \quad h^{\mu \nu}=\Lambda^{(\mu \nu)}
$$

of the metric. 
There is a certain ambiguity in the map $\sigma$ defined in (4.1). This must be so since over any associative algebra there are many differential calculi. As an example of this one can consider the case of constant commutators. The two key formulae are

$$
\theta^{\alpha}=\theta_{0}^{\alpha}-\epsilon \Lambda_{\beta}^{\alpha} \theta_{0}^{\beta}, \quad J^{\mu \nu}=J_{0}^{\mu \nu}
$$

The momenta are linear functions of the position and there is but one calculus based on the derivations associated to the momenta. It is given by the duality relations

$$
d x^{\mu}\left(e_{\alpha}\right)=\left[p_{\alpha}, x^{\mu}\right]=\delta_{\alpha}^{\mu}
$$

So amongst the set of differential calculi there is one which is based on the derivations defined by the momenta. This is the one which we define to be the image of $\sigma$.

Suppose we were to chose another 'nearby', based on the frame

$$
\theta^{\alpha}=d x^{\alpha}-\epsilon \Lambda_{\beta}^{\alpha} d x^{\beta}
$$

and defined by some matrix $\Lambda_{\beta}^{\alpha}$. We use the fact that the formulae of Section 3 remain valid but with the extra condition that $I=0$. In particular, from Equations (3.19) we find that the 2 -form $\hat{\Lambda}$ is a coboundary. It does not contribute to the Riemann tensor. So the perturbed differential calculus engenders a trivial perturbation of the metric. This result is difficult to understand intuitively since one would expect the metric components to change if the symmetric part $\Lambda_{(\alpha \beta)}$ of $\Lambda_{\alpha \beta}$ does not vanish. However the Jacobi identities force $\Lambda_{(\alpha \beta)}$ to be the symmetric gradient of a 1-form $A$; it therefore does not contribute to the Riemann tensor.

\section{Phase space}

It is obviously the case that in the commutative limit the 4 coordinate generators tend to the space-time coordinates and the 4 momenta tend to the conjugate momenta. The 8 generators become the coordinates of phase space. For this to be consistent all Jacobi identities must be satisfied, including those with two and three momenta. We consider first the identities

$$
\left[p_{\alpha},\left[p_{\beta}, x^{\mu}\right]\right]+\left[p_{\beta},\left[x^{\mu}, p_{\alpha}\right]\right]+\left[x^{\mu},\left[p_{\alpha}, p_{\beta}\right]\right]=0 .
$$

One easily see that, using the identities (3.14) and (3.17) as well as the assumption that the center is trivial we find that

$$
i \hbar\left[p_{\alpha}, p_{\beta}\right]=(K-\epsilon(\hat{\Lambda}-d A))_{\alpha \beta}=(K+\epsilon \hat{I})_{\alpha \beta}
$$

with

$$
K=-J_{0}^{-1}
$$

That is,

$$
i \hbar\left[p_{\alpha}, p_{\beta}\right]=-J_{\alpha \beta}^{-1}+o\left(\epsilon^{2}\right) .
$$


The remaining identities, involving only the momenta, are then satisfied by virtue of the fact that the 2-form $\hat{\Lambda}$ is closed. There is evidence to the fact that this relation is valid to all orders in $\epsilon$.

From the Jacobi identities we find that

$$
\left[p_{\alpha}-J_{0 \alpha \mu}^{-1} x^{\mu}, x^{\nu}\right]=\delta_{\alpha}^{\nu}-J_{0 \alpha \mu}^{-1}\left(J_{0}^{\mu \nu}+\epsilon I^{\mu \nu}\right)+\epsilon \Lambda_{\alpha}^{\nu}=\epsilon\left(\Lambda_{\alpha}^{\nu}-J_{0 \alpha \mu}^{-1} I^{\mu \nu}\right)=0 .
$$

For some set of constants $c_{\alpha}$ therefore, if the center of the algebra is trivial, we can write

$$
i k p_{\alpha}=J_{0 \alpha \mu}^{-1} x^{\mu}+c_{\alpha}
$$

The 'Fourier transform' is linear.

Let $J_{0}^{\mu \alpha}$ be an invertible matrix of real numbers. For each such matrix there is an obvious map from the algebra to the geometry given by

$$
J^{\mu \nu} \mapsto e_{\alpha}^{\nu}=J_{0 \alpha \mu}^{-1} J^{\mu \nu}
$$

For such frames we introduce momenta $p_{\alpha}$ and find that

$$
\left[p_{\alpha}, x^{\nu}\right]=e_{\alpha}^{\nu}=J_{0 \alpha \mu}^{-1} J^{\mu \nu}=(i \hbar)^{-1} J_{0 \alpha \mu}^{-1}\left[x^{\mu}, x^{\nu}\right]
$$

That is

$$
\left[i k p_{\alpha}-J_{0 \alpha \mu}^{-1} x^{\mu}, x^{\nu}\right]=0 .
$$

We can conclude therefore that (5.6) is satisfied. We can interpret the results of the previous section as the statement that this condition is stable under small perturbations of the geometry or algebra.

\section{An Example}

Consider $(2-d)$-Minkowski space with coordinates $(t, x)$ which satisfy the commutation relations $[t, x]=h t$ and with a geometry encoded in the frame $\theta^{1}=t^{-1} d x, \theta^{0}=t^{-1} d t$. These data describe [9] a noncommutative version of the Lobachevski plane. The region around the line $t=1$ can be considered as a vacuum. For the approximations of the previous section to be valid we must rescale $t$ so that in a singular limit the vacuum region becomes the entire space. We can do this by setting

$$
t=1+c t^{\prime}
$$

and consider the limit $c \rightarrow 0$. So that the geometry remain invariant we must scale the metric. We do this by rescaling $\theta^{0}$

$$
\theta^{0} \mapsto c^{-1} \theta^{0}
$$

The commutation relations become then

$$
\left[t^{\prime}, x\right]=c^{-1} h+h t^{\prime}
$$


and to leading order in $c$ the frame becomes

$$
\theta^{0}=\left(1-c t^{\prime}\right) d t^{\prime}, \quad \theta^{1}=\left(1-c t^{\prime}\right) d x
$$

From the definitions (3.2) we find that

$$
\begin{array}{ll}
J_{0}^{01}=c^{-1} h, & \epsilon I^{01}=h t^{\prime}, \\
J_{0,01}=-c h^{-1}, & \epsilon \Lambda_{\beta}^{\alpha}=c t^{\prime} \delta_{\beta}^{\alpha}
\end{array}
$$

and therefore we obtain the map $\sigma$ as defined in the previous section. This example is not quite satisfactory since the cocycle conditions (3.14) are vacuous in dimension two.

\section{The WKB Ansatz}

We now suppose that the algebra $\mathcal{A}$ is a tensor product

$$
\mathcal{A}=\mathcal{A}_{0} \otimes \mathcal{A}_{\omega}
$$

of a 'slowly-varying' factor $\mathcal{A}_{0}$ in which all amplitudes lie and a 'rapidly-varying' phase factor which is of order-of-magnitude $\epsilon$ so that only functions linear in this factor can appear. The generic element $f$ of the algebra is of the form then

$$
f\left(x^{\lambda}, \phi\right)=f_{0}\left(x^{\lambda}\right)+\epsilon f_{1}\left(x^{\lambda}\right) e^{i \omega \phi}
$$

Because of the condition on $\epsilon$ these elements form an algebra. We suppose that both $\Lambda$ and $I$ belong to $\mathcal{A}_{\omega}$. We introduce the normal $\xi_{\alpha}=e_{\alpha} \phi$ to the surfaces of constant phase. From (3.19) we find that

$$
\hat{\Lambda}_{\alpha \beta}^{-}=-\hat{I}_{\alpha \beta}+\xi_{[\alpha} A_{\beta]}
$$

The 2 -form $\hat{\Lambda}^{-}$is, to within a constant, a plane-wave-type solution to Maxwell's equations.

The expression for the metric becomes

$$
h^{\alpha \beta}=J_{0 \delta \gamma}^{-1} I^{\gamma(\alpha} \eta^{\beta) \delta}+\xi^{(\alpha} J_{0}^{\beta)} A^{\gamma} .
$$

The Riemann tensor in the limit we are considering, given by the expression

$$
R_{\alpha \beta \gamma \delta}=\frac{1}{4} \epsilon \xi_{[\alpha} \ddot{h}_{\beta][\gamma} \xi_{\delta]},
$$

depends only on the first term, linear in $I$. We have defined therefore a map

$$
I^{\alpha \beta} \mapsto R_{\beta \gamma \delta}^{\alpha}
$$

from the algebra to the geometry. Although there is a certain amount of ambiguity in the definition of the map as far as the components of the metric are concerned, this ambiguity drops from the curvature. In Section 4 we showed that all possible perturbations of the differential calculi, except for the one which we have chosen, leave the curvature invariant. 


\section{Dispersion relations}

The Ricci tensor for the perturbation $h_{\mu \nu}$ to a flat metric is given by

$$
R_{\alpha \beta}=-\frac{1}{4} \epsilon \omega^{2}\left(\xi^{2} h_{\alpha \beta}-\xi^{\gamma} h_{\gamma(\beta} \xi_{\alpha)}+h_{\gamma}^{\gamma} \xi_{\alpha} \xi_{\beta}\right)
$$

From Equation (7.4) we see then that it is a linear expression in the perturbation $I^{\mu \nu}$ to the commutation relations. The Einstein tensor for the perturbation is given by

$$
G_{\alpha \beta}=-\frac{1}{4} \epsilon \omega^{2}\left(\xi^{2} \bar{h}_{\alpha \beta}+\bar{h}_{\gamma \delta} \xi^{\gamma} \xi^{\delta} g_{\alpha \beta}-\xi^{\gamma} \bar{h}_{\gamma(\beta} \xi_{\alpha)}\right)
$$

in terms of

$$
\bar{h}_{\alpha \beta}=h_{\alpha \beta}-\frac{1}{2} h g_{\alpha \beta} .
$$

From Equation (7.4) we see then that both are linear expressions in the perturbation $I^{\mu \nu}$ to the commutation relations. The vacuum field equations are given by

$$
\xi^{2} \bar{h}_{\alpha \beta}-\xi^{\gamma} \bar{h}_{\gamma(\alpha} \xi_{\beta)}+\bar{h}_{\gamma \delta} \xi^{\gamma} \xi^{\delta} g_{\alpha \beta}=0 .
$$

We require a plane-wave-like solution to the condition (3.14), one which is not the differential of a 1-form. Wave-front surfaces are 2-surfaces and on such surfaces nontrivial 2 -forms can exist. This is however very formal since the surfaces in question are noncompact. Within the context of the WKB approximation one can distinguish between exact and non-exact closed 2-forms. If $\hat{I}$ has frame components

$$
\hat{I}_{\alpha \beta}=\hat{I}_{0 \alpha \beta} e^{i \omega \phi}
$$

then the differential has to leading order the components

$$
(d \hat{I})_{\alpha \beta \gamma}=i \omega\left(\xi_{\alpha} \hat{I}_{\beta \gamma}+\xi_{\beta} \hat{I}_{\gamma \alpha}+\xi_{\gamma} \hat{I}_{\alpha \beta}\right) .
$$

An example of a solution is

$$
\hat{I}_{\alpha \beta}=\frac{1}{2} \epsilon_{\alpha \beta \gamma \delta} \hat{I}^{* \gamma \delta}
$$

with

$$
\hat{I}^{* \gamma \delta} \xi_{\delta}=0 .
$$

It follows from (8.6) that if we multiply the cocycle condition $d \hat{I}=0$ by $\xi_{\alpha}$ we obtain

$$
\xi^{2} \hat{I}_{\alpha \beta}+\xi^{\gamma} \hat{I}_{\gamma[\alpha} \xi_{\beta]}=0
$$

This equation is very similar in structure to (8.4) and contains the essential information of the latter. From it one can read off the dispersion relations. One sees that either $\hat{I}$ is exact, that is the metric perturbation is non-radiative, or $\xi^{2}=0$. We discuss some of the details of this in the Appendix. 


\section{Nonlinearities}

Aided by a simplifying assumption, one can readily include the effects of the higherorder terms neglected in the previous calculations. Let $J^{\mu \nu}(z, \bar{z})$ be an arbitrary antisymmetric matrix whose elements belong to the subalgebra generated by two elements $z$ and $\bar{z}$ of four $x^{\mu}$. Suppose further that $[z, \bar{z}]=0$ so that the subalgebra is abelian and to be explicit suppose that $z=x^{3}+x^{0}$ and that $\bar{z}=x^{3}-x^{0}$. To be consistent then we must suppose further that $J^{03}=0$. Let $J^{\mu \alpha}$ be an arbitrary invertible matrix of complex numbers. Reality conditions, which we shall examine in more detail in a future publication force $J^{\mu \alpha}$ to be a real matrix to lowest order. We define the geometry such that

$$
\left[p_{\alpha}, x^{\mu}\right]=e_{\alpha}^{\mu}=J_{\alpha \sigma}^{-1} J^{\sigma \mu} .
$$

The notation is consistent since it follows that

$$
J^{\sigma \mu}=J^{\sigma \alpha} e_{\alpha}^{\mu}
$$

Define now the commutators to be

$$
\left[x^{\mu}, x^{\nu}\right]=i \hbar J^{\mu \nu}
$$

This will be consistent provided the Jacobi identities

$$
\epsilon_{\lambda \mu \nu \rho} J^{\lambda \alpha} e_{\alpha} J^{\mu \nu}=0
$$

are satisfied. We shall return to this equation later. It follows immediately that the Leibniz identities (2.3) are satisfied.

Define finally

$$
i \hbar\left[p_{\alpha}, p_{\beta}\right]=-J_{\alpha \mu}^{-1} e_{\beta}^{\mu} .
$$

The Lie algebra generated by the 8 elements is a consistent Lie algebra provided the initial Jacobi identities are satisfied. The same logic as that which lead to the dispersion relation in Section 8 leads here to the conclusion that the matrix of commutators must be a function only of $z$ (or $\bar{z}$ ) and that the normal to the surface $z=z_{0}$ must be a null vector. Under Wick rotation the matrix $J^{\mu \nu}$ would become a matrix of analytic functions.

\section{Recapitulation}

In previous publications [9] we have shown that to a noncommutative algebra defined by a commutator $J^{\mu \nu}$ and a differential calculus defined by a frame $\theta^{\alpha}$ one can associate (almost) a unique geometry defined by metric and connection. The question of exactly what part of the information in the curvature tensor comes from the commutator and which part from the frame remains open. One might conjecture that if 
the couple $\left(J^{\mu \nu}, \theta^{\alpha}\right)$ defines one geometry with curvature map $\operatorname{Curv}\left(J^{\mu \nu}, \theta^{\alpha}\right)$ and the couple $\left(J^{\mu \nu}, \theta^{\prime \alpha}\right)$ a second geometry with curvature map $\operatorname{Curv}\left(J^{\mu \nu}, \theta^{\prime \alpha}\right)$ then one has

$$
\operatorname{Curv}\left(J^{\mu \nu}, \theta^{\prime \alpha}\right)=\operatorname{Curv}\left(J^{\mu \nu}, \theta^{\alpha}\right) .
$$

There are counter-examples to this conjecture; it is not true.

A weaker conjecture is that (10.1) is valid if the second frame is a small perturbation

$$
\theta^{\prime \alpha}=\theta^{\alpha}-\epsilon \Lambda_{\beta}^{\alpha} \theta^{\beta}
$$

of the first. A yet weaker conjecture is that the equality (10.1) is valid if the commutator is constant $J^{\mu \nu}=J_{0}^{\mu \nu}$ and the initial frame is the exact frame $\theta^{\alpha}=d x^{\alpha}$. We have shown that this is in fact true. Furthermore we have shown that if the commutator is perturbed to $J^{\mu \nu}=J_{0}^{\mu \nu}+\epsilon I^{\mu \nu}$ and the frame $\theta^{\alpha}$ is consistently perturbed to $\theta^{\prime \alpha}$ then one has the equality

$$
\operatorname{Curv}\left(J^{\mu \nu}, \theta^{\prime \alpha}\right)=\operatorname{Curv}\left(I^{\mu \nu}\right) .
$$

In other words the perturbation of the Riemann map depends only on the perturbation $I$ of $J_{0}$ and not on its extension to the frame.

A second point which we have investigated is the status of the field equations. In the 'simplest' cases it would seem to be true that the frame is dual to a set of derivations $e_{\alpha}$ of the algebra and that these derivations are inner with associated momenta $p_{\alpha}$. It would seem then that the theory contains only four dynamical degrees of freedom. This is precisely the number of degrees of freedom of the conformal tensor (in dimension four). One could conjecture then that the Ricci tensor is fixed and calculable. We have shown this to be the case if the algebra is a high-frequency perturbation of a flat background.

We have derived a relation between the structure of an associated algebra as defined by the right-hand side $J$ of the commutation relations between the generators $x^{\mu}$ on the one hand and the metrics which the algebra can support, that is, which are consistent with the structure of a differential calculus over the algebra on the other. We have expressed this relation as the map (1.5) from the frame to $J$ which defines the algebra. The essential ingredients in the definition of the map are the Leibniz rules and the assumption (2.1) on the structure of the differential calculus. Although there have been found [16, 17, 4, 6] numerous particular examples, there is not yet a systematic discussion of either the range or kernel of the map. We have here to a certain extent alleviated this, but only in the context of perturbation theory around a vacuum and even then, only in the case of a high-frequency wave. A somewhat similar relation has been found [18] in the case of radiative, asymptotically-flat space-times.

\section{Conclusion}

We started with a consistent flat-space solution to the constraints of the algebra and of the geometry, a solution with the unusual property that its momenta and position 
stand in a relation of simple duality, a consequence of which is the fact that the Fourier-transformation is local. We then perturbed both structures, the geometric and the algebraic, in a seemingly arbitrary manner, but within the context of linearperturbation theory and requiring that the constraints remain valid. We were able to completely solve the constraints of the perturbation and exhibit a closed solution, which in the WKB situation, implied that the Ricci tensor was necessarily flat. However the seemingly general solutions we started with turned out, all of them, to satisfy the simple duality of the original solution, a fact which would tend to indicate that they were not really sufficiently general. So whereas at best have presented a solid indication that in the noncommutative context we have been persuing the Ricci tensor can be considered as calculable; at least we have indicated an interesting set of solutions to the algebro-geometic problem which have the duality property of the original flat-space.

\section{Appendix: WKB cohomology}

We briefly motivate here the notation used in Section 8 . We introduced the algebra of de Rham forms with a different differential inspired from the WKB approximation. The differential can be introduced for all forms but we give the construction only for the case of 2 -forms. Let $f_{\alpha \beta}$ be a 2 -form and define the differential $d_{\xi}$ of $f$ by the Formula (3.14). The interesting point is that the rank of the cohomology module $H^{2}$, an elementary form of Spencer cohomology, depends on the norm of $\xi$. Let $c$ be a 2-cocycle. Then

$$
\xi_{\alpha} c_{\beta \gamma}+\xi_{\beta} c_{\gamma \alpha}+\xi_{\gamma} c_{\alpha \beta}=0 .
$$

We multiply this by $\xi^{\alpha}$ to obtain the condition (8.9). There are two possibilities. If $\xi^{2} \neq 0$ then it follows immediately that the 2 -cocycle is exact. That is, $H^{2}=0$. If on the other hand $\xi^{2}=0$ then there are cocycles which are not exact. One can think of theses as plane-wave solutions to Maxwell's equations. We can reformulate the result of Section 8 as a statement of the dependence of the Riemann tensor uniquely on the cohomology:

$$
\operatorname{Curv}=\operatorname{Curv}\left[H^{2}\right]
$$

\section{Acknowledgment}

This article was started while JM was visiting the Humboldt Universität, Berlin and MB and he were visiting the A. Einstein Institut, Golm. They would like to thank D. Lüst as well as H. Nicolai and S. Theisen for their hospitality during this period. They would also like to thank J. Mourad and S. Waldmann for enlightening comments. The research was supported by the EPEAEK programs "Pythagoras" and jointly funded by the European Union (75\%) and the Hellenic state (25\%). The article assumed its present form while JM was visiting the W. Heisenberg Institut, München. He would like to thank F. Meyer and J. Wess for their hospitality during this period. 


\section{References}

[1] A. Connes, "The action functional in non-commutative geometry", Commun. Math. Phys. 117 (1988) 673.

[2] A. Connes, "Gravity coupled with matter and the foundation of non-commutative geometry", Commun. Math. Phys. 192 (1996) 155.

[3] J. Madore and J. Mourad, "Quantum space-time and classical gravity", J. Math. Phys. 39 (1998), no. 1, 423-442.

[4] M. Maceda, J. Madore, P. Manousselis, and G. Zoupanos, "Can noncommutativity resolve the Big-Bang singularity?", Euro. Phys. Jour. C 36 (2004) 529-534, hep-th/0306136.

[5] M. Burić, T. Grammatikopoulos, J. Madore, and G. Zoupanos, "On the fuzzy Kasner metric", (preprint) - (2005) -.

[6] M. Burić and J. Madore, "A dynamical 2-dimensional fuzzy space", Phys. Lett. B622 (2005) 183-191, hep-th/0507064.

[7] J. Madore, "Kaluza-Klein aspects of noncommutative geometry", in Differential Geometric Methods in Theoretical Physics, A. I. Solomon, ed., pp. 243-252. World Scientific Publishing, 1989. Chester, August 1988.

[8] J. Madore, "On the resolution of space-time singularities", Int. J. Mod. Phys. B 14 no. 22-23, 2419-2425. Torino, July 1999.

[9] J. Madore, An Introduction to Noncommutative Differential Geometry and its Physical Applications. No. 257 in London Mathematical Society Lecture Note Series. Cambridge University Press, second ed., 2000. 2nd revised printing.

[10] S. Doplicher, "Spacetime and fields, a quantum texture", in Jadczyk et. al. 19, pp. 204-213. hep-th/0105251. Karpacz, February 2001.

[11] M. Maceda and J. Madore, "On the resolution of space-time singularities II", J. Nonlin. Math. Phys. 11S1 21-36. hep-ph/0401055 Portoroz, Slovenia, July 2003.

[12] J. Madore and J. Mourad, "Algebraic Kaluza-Klein cosmology", Class. and Quant. Grav. 10 (1993), no. 10, 2157-2170.

[13] J. Madore, "The fuzzy sphere", Class. Quant. Grav. 9 (1992) 69-88.

[14] P. Aschieri, M. Dimitrijevic, F. Meyer, and J. Wess, "Noncommutative geometry and gravity", hep-th/0510059.

[15] E. Langmann and R. J. Szabo, "Teleparallel gravity and dimensional reductions of noncommutative gauge theory", Phys. Rev. D64 (2001) 104019, hep-th/0105094.

[16] G. Fiore, M. Maceda, and J. Madore, "The real Quantum Plane as part of 2d-Minkowski space", in Jadczyk et. al. 19. Karpacz, February 2001.

[17] B. L. Cerchiai, G. Fiore, and J. J. Madore, "Geometrical tools for Quantum Euclidean spaces", Commun. Math. Phys. 217 (2001), no. 3, 521-554, math.QA/0002007. 
[18] J. Madore, "On Poisson structure and curvature", in Coherent states, differential and quantum geometry, S. T. Ali, A. Odzijewicz, M. Schlichenmaier, and A. Strasburger, eds., vol. 43 of Rep. on Math. Phys., pp. 231-238. 1999. gr-qc/9705083. Bialowieza, July 1997.

[19] A. Jadczyk, J. Lukierski, and J. Rembielinski, eds., "New developments in fundamental interactions theories", Proceedings of XXXVII Karpacz Winter School of Theoretical Physics. Karpacz, February 2001. vol. 589 of AIP Conference Proceedings. American Institute of Physics, 2001. 